\title{
Aneurisma de aorta abdominal justa-renal: correção endovascular combinada com derivação ilíaco-renal direita para criar colo proximal adequado
}

\author{
Juxtarenal abdominal aortic aneurysm: combined endovascular \\ and open repair with right iliorenal bypass to create \\ adequate proximal neck
}

\author{
Matheus Bredarioli'1, Marcelo Bellini Dalio'1, Cleber Aparecido Pita Bezerra ${ }^{2}$, Carlos Eli Piccinato ${ }^{3}$, \\ Jesualdo Cherri ${ }^{4}$
}

\section{Resumo}

Homem de 78 anos de idade, portador de múltiplas morbidades clínicas deu entrada na emergência com um aneurisma de aorta abdominal justa-renal em condições hemodinâmicas estáveis. A tomografia computadorizada caracterizou aneurisma de $6 \mathrm{~cm}$ de diâmetro, e a distância do colo proximal do aneurisma era de $5 \mathrm{~mm}$ em relação à artéria renal direita e $28 \mathrm{~mm}$ à esquerda. Em virtude das condições clínicas do paciente, optou-se pelo reparo endovascular, mas previamente fez-se uma derivação ilíaco-renal direita com enxerto de politetrafluoretileno, via retroperitoneal para se criar um colo proximal adequado. Após quatro dias, o aneurisma de aorta abdominal foi corrigido colocando-se uma endoprótese Excluder ${ }^{\circledR}$ sem intercorrências. O seguimento pós-operatório evidenciou boa perfusão do rim esquerdo e ausência de migração ou endoleak da endoprótese. Este caso ilustra a combinação de técnicas para tornar possível o reparo de aneurisma de aorta abdominal justa-renal em pacientes de alto risco cirúrgico e anatomia desfavorável.

Palavras-chave: Aneurisma da aorta abdominal, cirurgia, prótese vascular, endovascular, artéria renal.

\section{Introdução}

Atualmente, o padrão-ouro para o tratamento do aneurisma de aorta abdominal (AAA) é ainda a operação convencional. O advento do reparo endovascular (EVAR), entretanto, trouxe grande benefício aos pacientes com múltiplas co-morbidades clínicas e risco

\begin{abstract}
A 78-year-old man with a juxtarenal abdominal aortic aneurysm and several comorbid conditions was admitted at the emergency room in hemodynamically stable conditions. Computed tomography revealed an aneurysm measuring $6 \mathrm{~cm}$ in diameter beginning $28 \mathrm{~mm}$ below the left renal artery and $5 \mathrm{~mm}$ below the right renal artery. Because of the patient's clinical status, a bypass from the right iliac artery to the right renal artery was performed through a retroperitoneal approach using a polytetrafluoroethylene vascular graft. Four days later, an endovascular aneurysm repair was successfully performed using an Excluder ${ }^{\circledR}$ stent-graft. Postoperative follow-up showed good left renal perfusion and no migration or endoleak. This case illustrates the effectiveness of combining open and endovascular techniques to repair juxtarenal abdominal aortic aneurysm in high-risk patients with unfavorable anatomy.
\end{abstract}

Keywords: Abdominal aortic aneurysm, surgery, vascular graft, endovascular, renal artery.

cirúrgico elevado, desde que apresentem anatomia compatível com as endopróteses disponíveis.

Para assegurar uma fixação proximal adequada da prótese, a maioria dos fabricantes preconiza um colo aórtico infra-renal de no mínimo $15 \mathrm{~mm}$. Pacientes

1. Cirurgiões vasculares e pós-graduandos, Área Clínica Cirúrgica, Departamento de Cirurgia e Anatomia, Faculdade de Medicina de Ribeirão Preto - Universidade de São Paulo (FMRP-USP), Ribeirão Preto, SP.

2. Médico residente, Disciplina de Cirurgia Vascular, Departamento de Cirurgia e Anatomia, FMRP-USP, Ribeirão Preto, SP.

3. Professor titular, Disciplina de Cirurgia Vascular, Departamento de Cirurgia e Anatomia, FMRP-USP, Ribeirão Preto, SP.

4. Professor associado, Disciplina de Cirurgia Vascular, Departamento de Cirurgia e Anatomia, FMRP-USP, Ribeirão Preto, SP.

Trabalho desenvolvido na Disciplina de Cirurgia Vascular do Departamento de Cirurgia e Anatomia da FMRP-USP e apresentado como pôster no VII SOBRICE, ocorrido em São Paulo, de 3 a 5 de novembro de 2005.

Artigo submetido em 08.03.07, aceito em 21.05.07. 
selecionados segundo estas recomendações evoluem com menores taxas de migração ou endoleak ${ }^{1}$. A implantação de endopróteses em AAA com colo infrarenal menor que $15 \mathrm{~mm}$, apesar de descrita com sucesso por vários autores, não é uma conduta unânime ${ }^{2}$. Em pacientes selecionados, é possível criar um colo proximal favorável por meio da revascularização cirúrgica da artéria renal.

Este relato descreve um caso de AAA cujo procedimento endovascular foi realizado após a criação de condições anatômicas favoráveis pela ampliação do comprimento do colo proximal por meio da realocação cirúrgica da artéria renal direita.

\section{Descrição do caso}

Homem de 78 anos deu entrada na emergência queixando-se de lombalgia. A tomografia computadorizada (TC) diagnosticou fratura de vértebra lombar com o achado de um AAA justa-renal com $6 \mathrm{~cm}$ em seu maior diâmetro transverso, sem sinais de rotura ou dissecção. A reconstrução tridimensional da TC mostrou que a artéria renal esquerda se originava a $28 \mathrm{~mm}$ acima do saco aneurismático, e a artéria renal direita apenas a $5 \mathrm{~mm}$ (Figura 1). Aparentemente, os dois rins tinham dimensões normais e excretavam contraste simetricamente. A história clínica do paciente incluía diabetes melito, hipertensão arterial sistêmica, coronariopatia compensada, leucemia mielóide monocítica crônica (síndrome mielodisplásica), doença pulmonar obstrutiva crônica, insuficiência renal crônica nãodialítica (creatinina $=2,5 \mathrm{mg} / \mathrm{dL})$ e obesidade $(102 \mathrm{~kg})$. O paciente foi encaminhado à disciplina de Cirurgia Vascular da FMRP-USP para planejamento de EVAR, já que ele não toleraria um clampeamento aórtico via aberta, devido a suas condições clínicas. Como ele apresentava um colo infra-renal curto do lado direito (5 $\mathrm{mm}$ ), optou-se por proceder uma abordagem aberta sem clampeamento aórtico antes do EVAR, com o objetivo de criar um colo proximal adequado. Através de um acesso retro-peritoneal direito, foi realizada uma derivação com politetrafluoretileno (PTFE) de $6 \mathrm{~mm}$, conectando a artéria ilíaca externa (anastomose látero- terminal) à artéria renal direita (anastomose términoterminal). O coto proximal da artéria renal direita foi ligado.

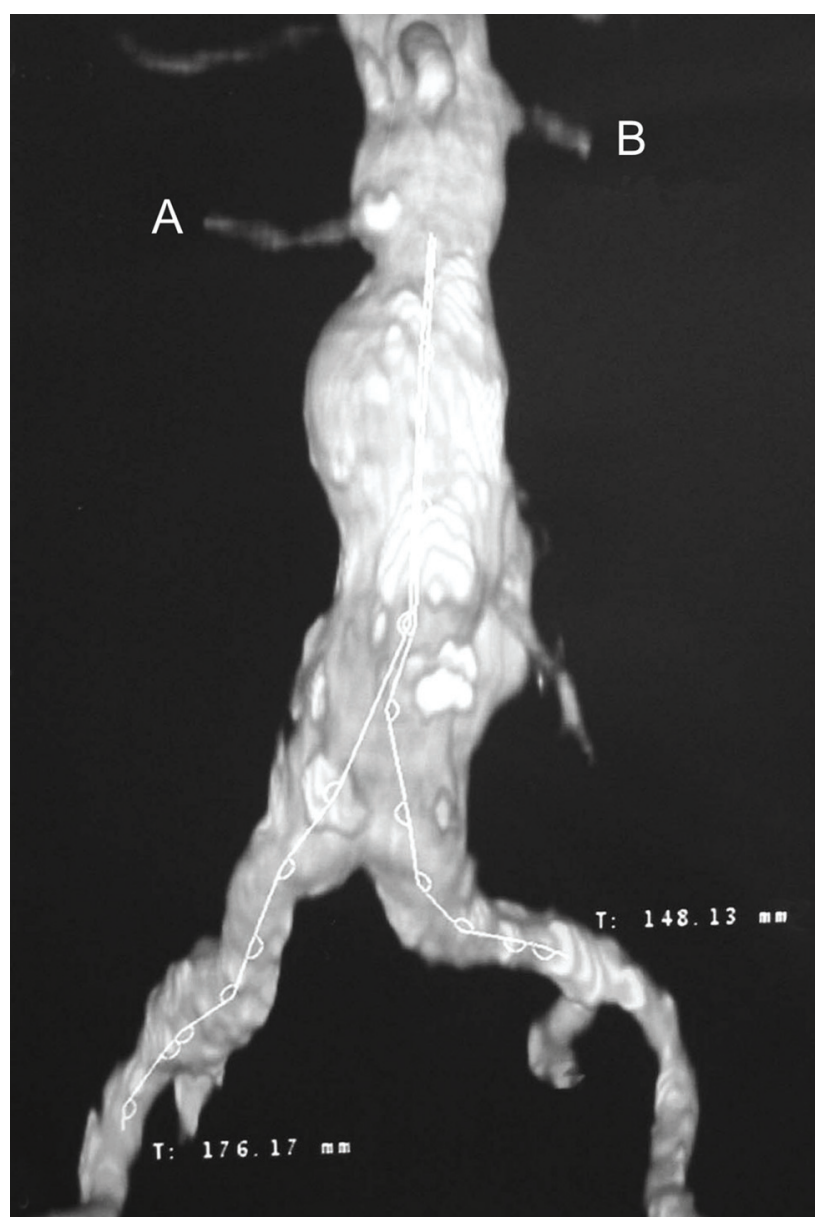

Figura 1 - Tomografia computadorizada (reconstrução tridimensional) mostrando AAA justa-renal com colo infra-renal de $5 \mathrm{~mm}$ em relação à artéria renal direita (A), e 28 mm em relação à esquerda (B)

Após o procedimento, o paciente foi levado à unidade de terapia intensiva (UTI). Quatro dias depois, foi executado EVAR com uma endoprótese Excluder $^{\circledR}$ (W.L. Gore, Inc, Flagstaff, Arizona, Estados Unidos), utilizando-se inguinotomia bilateral. A arteriografia final mostrou que a derivação ilíaco-renal estava patente e que a endoprótese estava bem posicionada e sem endoleak. Houve uma leve elevação na creatinina sérica, que retornou a valores inferiores aos do pré-operatório 
após alguns dias $(\mathrm{Cr}=1,5 \mathrm{mg} / \mathrm{dL})$. Em virtude de suas condições clínicas e da obesidade, a recuperação do paciente foi lenta e observaram-se complicações com a ferida operatória. Apesar disto, ele recebeu alta 12 dias após o segundo procedimento. A TC controle, um mês após, mostrou bom posicionamento da endoprótese e ausência de endoleak (Figura 2). A angiografia por ressonância magnética (A-IRM), utilizada no acompanhamento pós-operatório após 6 meses, revelou que a derivação ilíaco-renal direita estava patente e a perfusão renal estava normal. Não foi observado endoleak ou migração (Figura 3). No retorno do acompanhamento anual, o paciente estava totalmente recuperado do procedimento. Sua nova A-IRM não mostrou alterações, em comparação com a anterior.

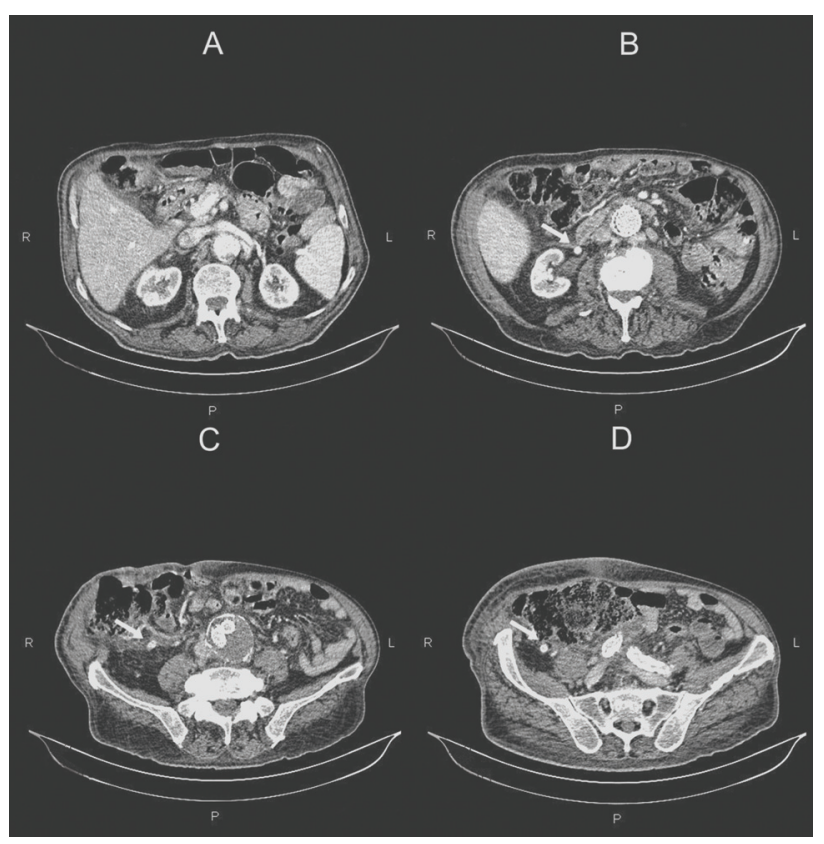

Figura 2 - Tomografia axial um mês após o procedimento mostrando perfusão renal simétrica (A), posicionamento adequado da endoprótese com ausência de endoleak (B,C), e posicionamento distal correto (D). Setas indicam derivação ilíaco-renal direita patente

\section{Discussão}

O conceito do reparo combinado aberto e endovascular não é novo. Na literatura, há relatos descrevendo EVAR associado à revascularização visceral no trata-

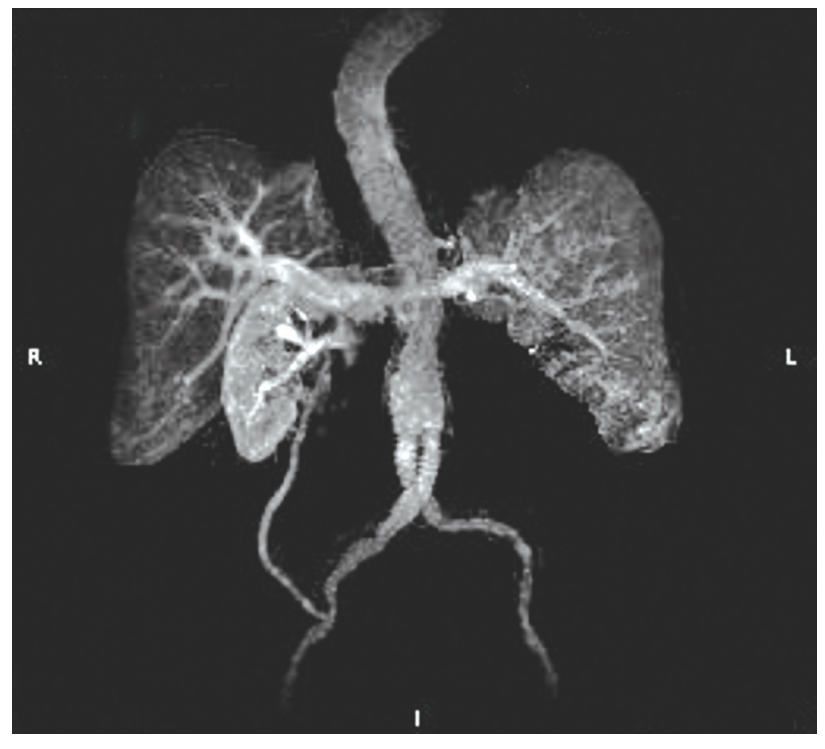

Figura 3 - Angiografia por ressonância magnética seis meses após o procedimento mostrando exclusão do aneurisma de aorta abdominal, ausência de endoleak, perviedade da derivação ilíaco-renal direita e perfusão renal adequada

mento de AAA infra ${ }^{3}$ e supra-renais ${ }^{4,5}$. O princípio teórico em que esta técnica se embasa é a associação das vantagens das duas modalidades de reparo, permitindo assim o tratamento de pacientes de alto risco, como aqui apresentado.

O reparo aberto, que ainda é o padrão-ouro, requer clampeamento aórtico, o que poderia provocar complicações fatais em pacientes de alto risco. Parodi et al. ${ }^{6}$ introduziram a técnica EVAR nos anos 80 , tendo como a principal vantagem evitar o clampeamento aórtico. No entanto, para que as endopróteses sejam liberadas com segurança e com o mínimo de complicações, certas condições anatômicas são preconizadas pelos fabricantes, incluindo a presença de um colo infra-renal de, no mínimo, $15 \mathrm{~mm}$. Com o objetivo de tornar o EVAR seguro em pacientes com colo infra-renal curto, novas endopróteses vem sendo estudadas e testadas ${ }^{7}$. Entretanto, a presença de um colo infra-renal de no mínimo $15 \mathrm{~mm}$ ainda é pré-requisito para a maioria dos produtos. Colos curtos são associados a complicações como migração e endoleak tipo $\mathrm{I}^{8}$.

O paciente em questão tinha um AAA com $6 \mathrm{~cm}$ de diâmetro, o que justifica seu reparo. O clampeamento 
aórtico supra-renal não seria tolerado em virtude de suas múltiplas co-morbidades, motivo pelo qual o procedimento cirúrgico foi contra-indicado. O EVAR isolado não seria aconselhável em virtude do colo infra-renal inadequado. A solução foi criar um colo proximal adequado por meio da revascularização cirúrgica da artéria renal direita com uma derivação ilíacorenal com PTFE. A oclusão da artéria renal direita não foi considerada, devido ao quadro de insuficiência renal com rins simétricos.

Escolheu-se o acesso retro-peritoneal direito para o procedimento de derivação por este proporcionar adequada exposição dos vasos renais e ilíacos e evitar manipulação de alças intestinais. Utilizou-se a artéria ilíaca externa direita como artéria doadora na derivação, para evitar o clampeamento aórtico e deixar as artérias ilíacas comuns livres para o ancoramento distal da endoprótese. O procedimento total foi realizado em duas intervenções separadas para evitar os efeitos adicionais do clampeamento renal direito e a infusão de contraste iodado num paciente com insuficiência renal crônica.

Observou-se, nos primeiros dias após os procedimentos, aumento dos níveis de creatinina sérica do paciente, que baixaram após hidratação adequada na UTI. Alguma dificuldade foi observada durante o EVAR pela presença da imagem de gás em alças intestinais à fluoroscopia, em virtude do íleo adinâmico. Apesar desta dificuldade, a endoprótese ficou bem posicionada sem imagem sugestiva de endoleak. No $30^{\circ}$ dia pós-operatório, a TC revelou bom posicionamento da endoprótese e ausência de endoleak. Seis meses e um ano após o procedimento, optou-se pelo seguimento com A-IRM, com o objetivo de evitar contraste iodado.

Uma tecnologia emergente no tratamento dos AAA justa-renais é o uso de endopróteses com ramificações e fenestrações para as artérias viscerais ${ }^{9}$. Há vários relatos na literatura descrevendo experiências bem sucedidas de casos isolados com esta nova tecnologia ${ }^{10,11}$, mas estudos de seguimento a longo prazo são necessários para a sua consolidação.
Em resumo, este caso ilustra como se pode combinar as técnicas cirúrgica e endovascular para tornar possível o tratamento de AAA justa-renal em pacientes de alto risco operatório e com anatomia desfavorável. Para o sucesso desta combinação, o cirurgião vascular deve dominar ambas as técnicas.

\section{Referências}

1. Dillavou ED, Muluk SC, Rhee RY, et al. Does hostile neck anatomy preclude successful endovascular aortic aneurysm repair? J Vasc Surg. 2003;38:657-63.

2. Mendonça CT, Moreira RCR, Timi JRR, et al. Comparação entre os tratamentos aberto e endovascular dos aneurismas da aorta abdominal em pacientes de alto risco cirúrgico. J Vasc Bras. 2005;4:232-42.

3. Lin PH, Madsen K, Bush RL, Lumsden AB. Iliorenal artery bypass grafting to facilitate endovascular abdominal aortic aneurysm repair. J Vasc Surg. 2003;38:183-5.

4. Kotsis T, Scharrer-Pamler R, Kapfer X, et al. Treatment of thoracoabdominal aortic aneurysms with a combined endovascular and surgical approach. Int Angiol. 2003;22:125-33.

5. Flye MW, Choi ET, Sanchez LA, et al. Retrograde visceral vessel revascularization followed by endovascular aneurysm exclusion as an alternative to open surgical repair of thoracoabdominal aortic aneurysm. J Vasc Surg. 2004;39:454-8.

6. Parodi JC, Palmaz JC, Barone HD. Transfemoral intraluminal graft implantation for abdominal aortic aneurysms. Ann Vasc Surg. 1991;5:491-9.

7. Chuter TA, Parodi JC, Lawrence-Brown M. Management of abdominal aortic aneurysm: a decade of progress. J Endovasc Ther. 2004;11 Suppl 2:II82-95.

8. Veith FJ, Baum RA, Ohki T, et al. Nature and significance of endoleaks and endotension: summary of opinions expressed at an international conference. J Vasc Surg. 2002;35:1029-35.

9. Linsen MA, Vos AW, Diks J, Rauwerda JA, Wisselink W. Fenestrated and branched endografts: assessment of proximal aortic neck fixation. J Endovasc Ther. 2005;12:647-53.

10. Greenberg RK, Haulon S, O'Neill S, Lyden S, Ouriel K. Primary endovascular repair of juxtarenal aneurysms with fenestrated endovascular grafting. Eur J Vasc Endovasc Surg. 2004;27:484-91.

11. Greenberg RK, Haulon S, Lyden SP, et al. Endovascular management of juxtarenal aneurysms with fenestrated endovascular grafting. J Vasc Surg. 2004;39:279-87.

Correspondência

Carlos Eli Piccinato

Av. Bandeirantes, 3900

CEP 14049-990 - Ribeirão Preto, SP

(16) 3633.0836

E-mail: cepiccin@fmrp.usp.br 